\title{
Variation in Individual Responses to Time-Restricted Feeding and Resistance Training
}

\author{
GRANT M. TINSLEY, JEFFREY S. FORSSE, GRANT B. MORGAN, NATALIE K. \\ BUTLER, ANNIE A. BANE, PAUL M. LA BOUNTY, and PETER W. GRANDJEAN
}

Baylor Laboratories for Exercise Science \& Technology; Department of Health, Human Performance, and Recreation; Baylor University; Waco, TX

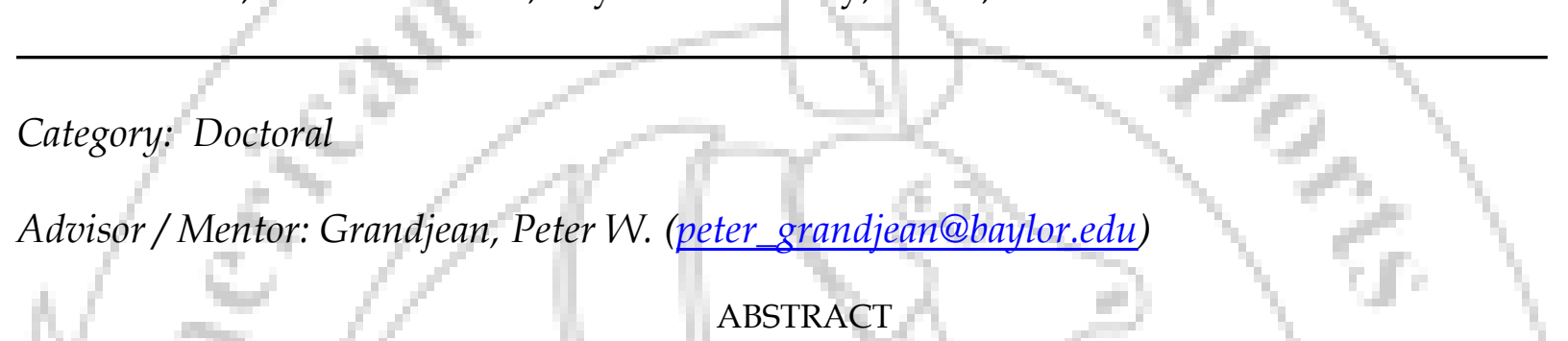

Time-restricted feeding (TRF) is a form of intermittent fasting which limits all caloric intake to a certain period of time each day in an attempt to reduce daily energy intake, promote weight loss, and improve health. Resistance training (RT) has been reported to increase muscular strength and improve body composition. Very limited information is available on the combination of TRF and RT. The purpose of this study was to examine the variation in individual body composition, dietary intake, and muscular performance responses to an 8-wk TRF and RT program. Healthy males $(n=20$; age $=22 \pm 3 y$; BMI $=27 \pm$ $6 \mathrm{~kg} / \mathrm{m} 2 ; \%$ fat $=22 \pm 6 \% \mathrm{wt}$ ) were randomized to TRF $+\mathrm{RT}$ or RT alone for $8 \mathrm{wks}$. RT was performed 3 dys/wk and consisted of alternate workouts of upper and lower body using a resistance progression scheme. TRF limited energy intake to a $4-\mathrm{hr}$ period on the 4 dys/wk when RT was not performed. Energy intake was not restricted in either group, and eating times were not specified in the RT alone group. Body composition, muscular performance, and dietary records were assessed at 0,4 , and 8 wks. Inter- and intraindividual variations in outcome measures were estimated by hierarchical linear growth modeling. The amount of variability attributable to characteristics between or within participants was evaluated from variance estimates. For TRF $+\mathrm{RT}$, percent changes ranged from -5.5 to $+2.6 \%$ for body weight, -22.1 to $+9.4 \%$ for fat mass, -7.7 to $+4.6 \%$ for lean body mass, +3.4 to $+30.4 \%$ for bench press $1-\mathrm{RM}$, and +10.1 to $+67.6 \%$ for leg press 1-RM. For RT alone, percent changes ranged from -6.6 to $+2.1 \%$ for body weight, -14.4 to $+12.6 \%$ for fat mass, -4.1 to $+3.9 \%$ for lean body mass, +4.9 to $+12.9 \%$ for bench press $1-\mathrm{RM}$, and +14.3 to $+37.7 \%$ for leg press 1-RM. Percentages of total variability attributed to inter-individual factors ranged from 3.3 to $49.2 \%$ for dietary measures, 59.0 to $93.9 \%$ for muscular performance, and 97.0 to $99.6 \%$ for body composition. Remaining variability was attributed to intra-individual factors. Individual responses to the study interventions varied widely. Differences between individuals were an important source of variability, indicating participant samples should be homogenous and/or quite large to examine changes in body composition or muscular performance using nutrition and exercise interventions. 